The counting of groups of our class of order $p^{12}$ by this method leads to the classification of similar pairs of matrices, an unsolved problem. The reader may check that our problem for two generators is easy, whereas for four generators seems unmanageable.

\title{
REFERENCES
}

1. G. Higman, Enumerating p-groups, I: Inequalities, Proc. London Math. Soc. vol. 10 (1960) pp. 24-30.

2. - Enumerating p-groups, II: Problems whose solution is PORC, Proc. London Math. Soc. vol. 10 (1960) pp. 566-582.

3. M. Hall, Jr., The theory of groups, New York, Macmillan, 1959.

4. K. W. Gruenberg, Residual properties of infinite soluble groups, Proc. London Math. Soc. vol. 7 (1957) pp. 29-62.

SOUTHERN ILLINOIS UNIVERSITY AND

The University of Chicago

\section{AN INEQUALITY FOR HERMITE POLYNOMIALS}

\section{JACK INDRITZ}

It is known that the Hermite polynomials

$$
H_{n}(x)=(-1)^{n} e^{x^{2}} \frac{d^{n}}{d x^{n}} e^{-x^{2}}, \quad n=0,1, \cdots,-\infty<x<\infty
$$

satisfy an inequality

$$
H_{n}(x) \leqq c\left(2^{n} n !\right)^{1 / 2} e^{x^{2} / 2}, \quad c \text { constant. }
$$

Erdelyi [1] states that $c=1.086435$ will serve. It has been conjectured that the best value for $c$ is 1 , and it is the purpose of this note to prove the conjecture.

As a basis for the discussion, we assume as known the following properties:

(3) $H_{n}(x)$ is a polynomial of degree $n$, having $n$ distinct real zeros. The coefficient of $x^{n}$ is $2^{n}$.

(4) $H_{n+1}(x)=2 x H_{n}(x)-H_{n}^{\prime}(x)$,

(5) $\quad H_{n}(x)-2 x H_{n-1}(x)+2(n-1) H_{n-2}(x)=0$, $n \geqq 2$,

(6) $H_{n}^{\prime \prime}(x)-2 x H_{n}^{\prime}(x)+2 n H_{n}(x)=0$,

(7) $H_{n}(-x)=(-1)^{n} H_{n}(x)$,

(8) $\quad H_{n}^{\prime}(x)=2 n H_{n-1}(x)$.

Received by the editors October 28, 1960. 
It will be useful to study the zeros of the normalized Hermite functions (see Hille [2]), defined by

$$
E_{n}(x)=\left(\pi^{1 / 2} 2^{n} n !\right)^{-1 / 2} e^{-x^{2} / 2} H_{n}(x), \quad n=0,1, \cdots
$$

The zeros of $E_{n}$ are those of $H_{n}$ and are distinct. In view of (3) it is seen that $H_{n}$ and hence $E_{n}$ is positive for sufficiently large positive $x$ while

$$
E_{n}^{\prime}(x)=\left(\pi^{1 / 2} 2^{n} n !\right)^{-1 / 2} e^{-x^{2} / 2}\left(H_{n}^{\prime}-x H_{n}\right)
$$

is negative for sufficiently large positive $x$. A consideration of the graph of $E_{n}$ then indicates $E_{n}^{\prime}$ must have $n+1$ distinct zeros, all real, with a zero of $E_{n}$ between two zeros of $E_{n}^{\prime}$.

In view of (8) and (9), we obtain, for $n \geqq 1$,

$$
\begin{aligned}
x E_{n} & =(2 n)^{1 / 2} E_{n-1}-E_{n}^{\prime}, \\
(2 n)^{1 / 2} E_{n} & =x E_{n-1}-E_{n-1}^{\prime},
\end{aligned}
$$

and from these

$$
\begin{aligned}
(2 n)^{1 / 2} E_{n}^{\prime} & =\left(2 n-x^{2}\right) E_{n-1}+x E_{n-1}^{\prime}, \\
(2 n)^{1 / 2} E_{n-1}^{\prime} & =x E_{n}^{\prime}-\left(2 n-x^{2}\right) E_{n} \\
\left(2 n-x^{2}\right) E_{n}^{2}+\left(E_{n}^{\prime}\right)^{2} & =\left(2(n-1)-x^{2}\right) E_{n-1}^{2}+\left(E_{n-1}^{\prime}\right)^{2}+2 E_{n-1}^{2}
\end{aligned}
$$

Equation (14) implies

$$
\left(2 n-x^{2}\right) E_{n}^{2}+\left(E_{n}^{\prime}\right)^{2}=2 \sum_{j=0}^{n-1} E_{j}^{2}
$$

and hence all zeros of $E_{n}^{\prime}$ lie in the interval $|x|<(2 n)^{1 / 2}$. Moreover, the zeros of $E_{n}$ are also in this interval.

Now the relative maxima of $\left|E_{n}(x)\right|, x \geqq 0$, steadily increase, i.e., if $x_{1}<x_{2}<\cdots<x_{j}$ are the non-negative zeros of $E_{n}^{\prime}$ for fixed $n$, then

$$
\left|E_{n}\left(x_{1}\right)\right|<\left|E_{n}\left(x_{2}\right)\right|<\cdots<\left|E_{n}\left(x_{j}\right)\right| .
$$

This is proved by showing that the derivative of

$$
g(x)=E_{n}(x)^{2}+\frac{E_{n}^{\prime}(x)^{2}}{2 n+1-x^{2}}, \quad|x|<(2 n+1)^{1 / 2},
$$

is positive between the zeros of $E_{n}{ }^{\prime}$. One uses here the fact that the differential equation satisfied by $E_{n}$ is

$$
E_{n}^{\prime \prime}(x)+\left(2 n+1-x^{2}\right) E_{n}(x)=0 .
$$

If $x_{0} \geqq 0$ is the point where $E_{n-1}(x), n \geqq 1$, achieves its maximum 
value, then (12) implies $E_{n}^{\prime}\left(x_{0}\right)>0$. This means that if $y_{0} \geqq 0$ is the point where $E_{n}$ achieves its maximum, we must have $y_{0}>x_{0}$. We shall show $E_{n}^{\prime}$ has no zero on the interval $x_{0} \leqq x<y_{0}$. Indeed, if the contrary were assumed, define

$$
h(x)=E_{n}^{2}+\frac{\left(E_{n}^{\prime}\right)^{2}}{2 n-x^{2}}=E_{n-1}^{2}+\frac{\left(E_{n-1}^{\prime}\right)^{2}}{2 n-x^{2}}, \quad x^{2}<2 n
$$

(the equality is a consequence of (14)), and note

$$
h^{\prime}(x)=\frac{2(2 n)^{1 / 2}}{\left(2 n-x^{2}\right)^{2}} E_{n}^{\prime} E_{n-1}^{\prime} .
$$

If $z_{0}$ were the largest zero of $E_{n}^{\prime}$ on $x_{0} \leqq x<y_{0}$, then $E_{n}^{\prime}$ is $\geqq 0$ on $z_{0} \leqq x \leqq y_{0}$, while $E_{n-1}^{\prime}$ is $\leqq 0$ on this interval, so that $h^{\prime}(x) \leqq 0$ on $z_{0} \leqq x \leqq y_{0}$, or

$$
E_{n}^{2}\left(z_{0}\right)=h\left(z_{0}\right) \geqq h\left(y_{0}\right)=E_{n}^{2}\left(y_{0}\right)
$$

contradicting (16). It follows that $E_{n}^{\prime}$ has no zero on the interval $x_{0} \leqq x<y_{0}$. But then

$$
h^{\prime}(x) \leqq 0, \quad x_{0} \leqq x \leqq y_{0},
$$

and so

$$
h\left(x_{0}\right)=E_{n-1}^{2}\left(x_{0}\right) \geqq h\left(y_{0}\right)=E_{n}^{2}\left(y_{0}\right) .
$$

This means

$$
\begin{gathered}
\max \left|E_{n}(x)\right| \leqq \max \left|E_{0}(x)\right|=\pi^{-1 / 4} \\
\left|H_{n}(x)\right| \leqq\left(2^{n} n !\right)^{1 / 2} e^{x^{2 / 2}} .
\end{gathered}
$$

Equality occurs for $x=0, n=0$.

\section{REFERENCES}

1. A. Erdelyi, W. Magnus, F. Oberhettinger and F. Tricomi, Higher transcendental functions, vol. 2, New York, McGraw-Hill, 1953, p. 208.

2. E. Hille, Reciprocal functions, Ann. of Math. vol. 26-27 (1924-26) pp. 427-464.

UNIVERSITY OF MinNESOTA 